\title{
Review Article \\ Pancreaticobiliary Maljunction Is Associated with Common Bile Duct Carcinoma: A Meta-Analysis
}

\author{
Yang Li, Jun Wei, Zhongxin Zhao, Tiangeng You, and Mingan Zhong \\ Department of Gastrointestinal Surgery, East Hospital, Tongji University, 150 Jimo Road, Pudong New District, Shanghai 200120, China \\ Correspondence should be addressed to Zhongxin Zhao; zhongxinzhao1999@hotmail.com
}

Received 15 August 2013; Accepted 3 October 2013

Academic Editors: G. Bocci and T. Nakanishi

Copyright (C) 2013 Yang Li et al. This is an open access article distributed under the Creative Commons Attribution License, which permits unrestricted use, distribution, and reproduction in any medium, provided the original work is properly cited.

\begin{abstract}
Objective. Pancreaticobiliary maljunction (PBM) has been reported to be associated with an increased risk of gallbladder carcinoma. However, the relationship between PBM and common bile duct carcinoma (CBDC) remains unclear. We aimed to conduct a metaanalysis to determine the available evidence on the association between PBM and CBDC. Methods. The pooled odds ratio (OR) and standard mean differences (SMD) with 95\% confidence interval (95\% CI) were used to estimate the effects. Results. A total of eight case-control studies and two cohort studies were identified. The incidence of PBM was higher in CBDC patients than in controls $(\mathrm{OR}=1.45 ; 95 \% \mathrm{CI}, 1.19-1.76)$. Compared with patients without PBM, CBDC patients with PBM were younger at the diagnosis age (SMD $=-0.46 ; 95 \% \mathrm{CI},-0.64$ to -0.28 ). A difference in the incidence of associated CDC was found between CBDC patients with or without PBM $(\mathrm{OR}=2.14 ; 95 \% \mathrm{CI}, 1.60-2.87)$. Conclusions. Compared with benign biliary tract diseases, the incidence of $\mathrm{PBM}$ was higher in CBDC patients, especially in relatively young patients. We also found that the incidence of CDC was higher in CBDC patients with PBM. These findings showed positive association between PBM and CBDC, which may help in identifying high-risk individuals.
\end{abstract}

\section{Introduction}

Common bile duct carcinoma (CBDC) is a malignancy arising from the ductular epithelium of the biliary tree, mainly located in the distal common bile duct, which accounts for $20-30 \%$ of all bile duct carcinomas (BDC) [1]. CBDC accounts for $1 \%$ of all gastrointestinal cancers [2]. But, it is more prevalent in Eastern Asian countries, including China $[1,3,4]$. The peak age for patients with CBDC is the seventh decade and the sex incidence shows a slight male preponderance $[5,6]$. The five-year survival rate of CBDC patients following surgical resection is approximately $5-15 \%[6,7]$. These figures represent the aggregate prognosis of all patients with CBDC [5-7]. Therefore, it is important to identify the high-risk group for $\mathrm{CBDC}$, especially in high-incidence countries, such as Japan, Korea, China, and Pakistan.

It is widely known that pancreaticobiliary maljunction (PBM) is an anomalous arrangement of pancreaticobiliary ductal system with the union of the pancreatic and biliary ducts locating outside the duodenal wall [8-11]. As the action of the sphincter muscle does not functionally affect the union, two-way regurgitation (pancreaticobiliary and biliopancreatic reflux) occurs, resulting in various pathological conditions in the biliary tract and pancreas $[9,12-15]$. Hasumi et al. [16] revealed that the incidence of PBM was 3.3\% among 12,399 patients who underwent hepatobiliary tract surgery and approximately $4.4 \%$ of the common bile duct cancer patients had PBM. Therefore, the positive correlation between PBM and CBDC has drawn increasing attention $[17,18]$.

Kamisawa et al. [12] collaborating with Working Committee of Clinical Practice Guidelines for Pancreaticobiliary Maljunction divided PBM into three distinct types based on imaging findings: (1) bile duct (junction) type, in which the bile duct joins the pancreatic duct at a right angle; (2) pancreatic duct (junction) type, in which the pancreatic duct joins the bile duct at an acute angle; (3) complex type, in 
which the two ducts meet in such a complex manner that the junction cannot be classified as either of the previous two types.

To elucidate the effect of PBM on the development of CBDC, we thus carried out a comprehensive meta-analysis of the current epidemiological literature to investigate the associations between the two diseases.

\section{Methods}

2.1. Literature Sources and Searches. Bibliography search was carried out in PubMed (1970 to December 2012) and Web of Science (1986 to December 2012). All aspects of the Preferred Reporting Items for Systematic Reviews and Meta-Analysis (PRISMA) statement were followed [19]. The medical subject heading $(\mathrm{MeSH})$ terms and key words used in the search included "pancreaticobiliary maljunction," "anomalous pancreaticobiliary ductal junction," "anomalous pancreaticobiliary ductal union" combined with "bile duct carcinoma(s)," or "bile duct cancer(s)," or "bile duct tumor(s)," or "bile duct neoplasm(s)," or "bile duct malignancy(ies)."

2.2. Study Selection. We included studies that met the following criteria: (i) case-control and cohort studies published in English; (ii) PBM as one of the exposures of interest and CBDC as one of the outcomes of interest (iii) conformation of PBM by endoscopic retrograde cholangiopancreatography (ERCP) or percutaneous transhepatic cholangiography (PTC) or magnetic resonance cholangiopancreatography (MRCP); (iv) diagnosis of CBDC based on surgery or pathology; and (v) reporting one of the following clinical data in patients with CBDC combined with PBM: age, sex, associated CDC, and type of PBM.

2.3. Data Extraction. The parameters from studies were extracted and entered into a database. The following data were collected: first author, year of publication, study design, country of origin, number of participants (cases and controls), potential confounders, diagnostic methods for PBM and bile duct carcinoma, and clinical data on patients with bile duct carcinoma associated with PBM, OR, and 95\% confidence interval (CI). The authors were contacted for additional information, when necessary. Data were independently extracted and analyzed by two researchers (Yang Li and Zhongxin Zhao) and final decision was reached by consensus, referring back to the original article.

2.4. Statistical Analysis. Summary OR estimates with their corresponding 95\% CI were calculated with a fixed-effects or random-effects model. Heterogeneity across studies was tested with the $Q$ and $I^{2}$ statistics. For the $Q$ statistic, the results were defined heterogeneous for $P<0.10$. A randomeffects model was applied in the occurrence of significant heterogeneity $(P<0.10)$. For $I^{2}$, a value of more than $50 \%$ was considered a measure of severe heterogeneity [20]. We conducted analyses stratified by study design, diagnosis age, and the incidence of CDC. Publication bias was assessed by Begg's funnel plot and Egger's test; the former was based on

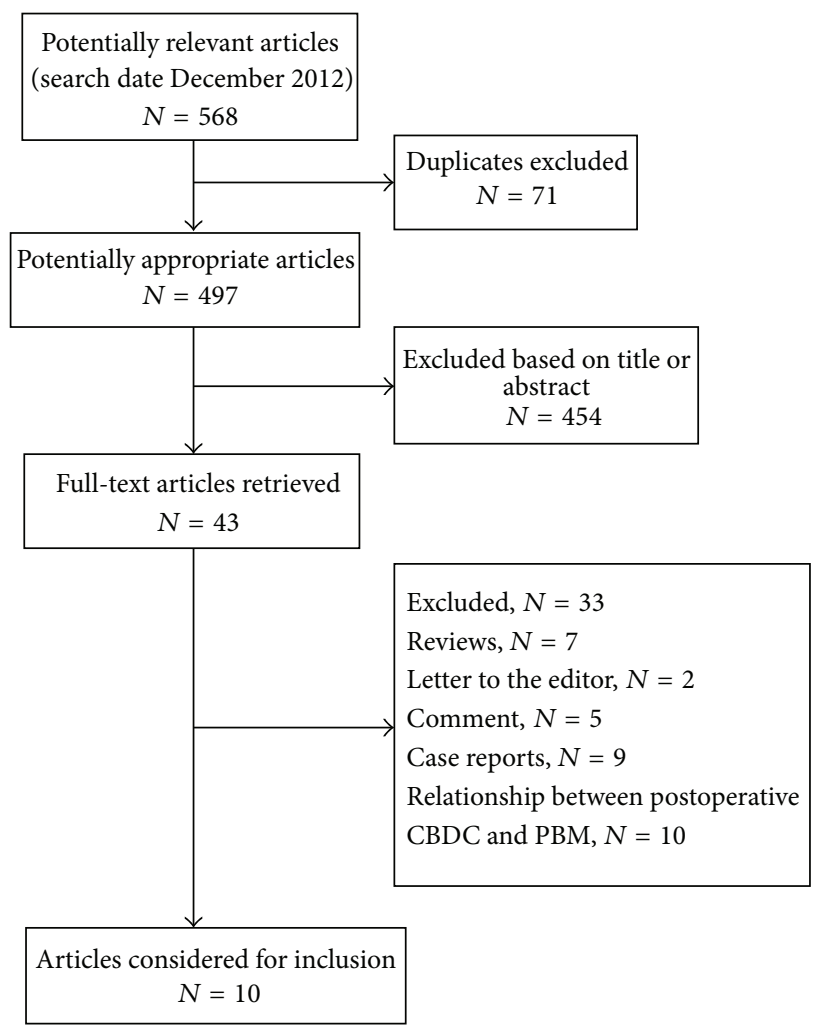

FIgURE 1: Article search flow chart showing the inclusion and exclusion of studies.

adjusted rank correlation and the latter on a regression model $(P<0.10$ as an indication for publication bias) [21, 22]. All statistical analyses were performed with STATA, version 11.0 (STATA, Corp., College Station, Lakeway Drive, TX, USA).

\section{Results}

3.1. Search Results. 568 articles were identified and screened by querying PubMed and Web of Science through December 2012, of which 71 were duplicates (Figure 1). Most of them $(n=454)$ were not focused on the relation between PBM and common bile duct carcinoma and were therefore not considered. Of the remaining 43 articles, 33 did not meet the inclusion criteria. Thus, the present analyses were based on 8 case-control studies and 2 cohort studies. A total of 2139 incident CBDC cases (144 incident PBM and 1995 incident without PBM) and 23,967 incident nonmalignant pancreaticobiliary diseases were included in this meta-analysis.

3.2. Characteristics of Eligible Studies. The characteristics of these studies were shown in Table 1 . Ten clinical trials $[8$, $10,13,16,18,23-27$ ] were identified to give the information with the incidence of PBM in CBDC patients and controls according to the inclusion criteria in the meta-analysis. After the communication with authors of the above studies, some information remains unclear with objective reasons. Wang et al. [23] and Suda et al. [26] did not provide the diagnosis age of CBDC patients with or without PBM. Suda et al. [26] 


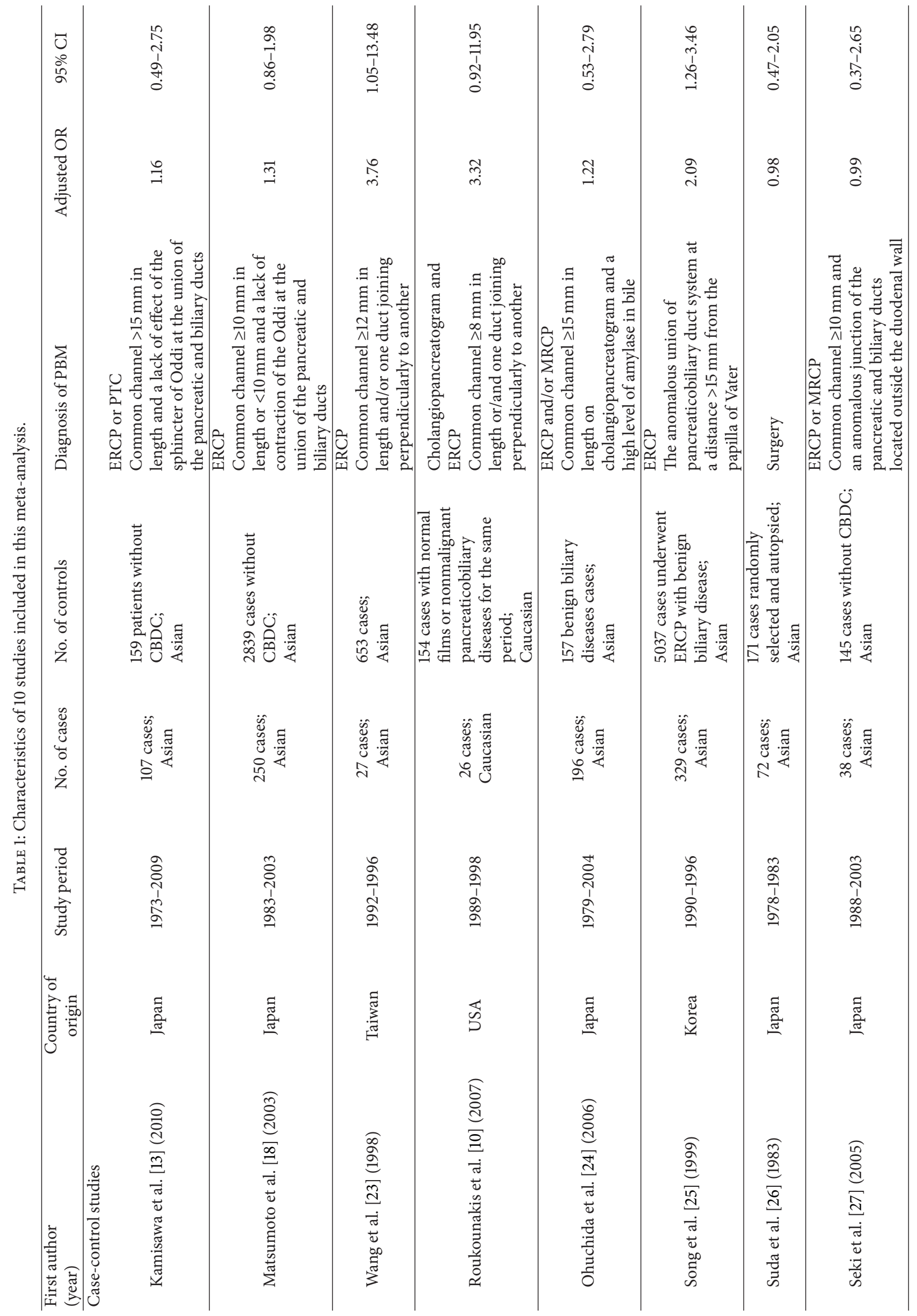




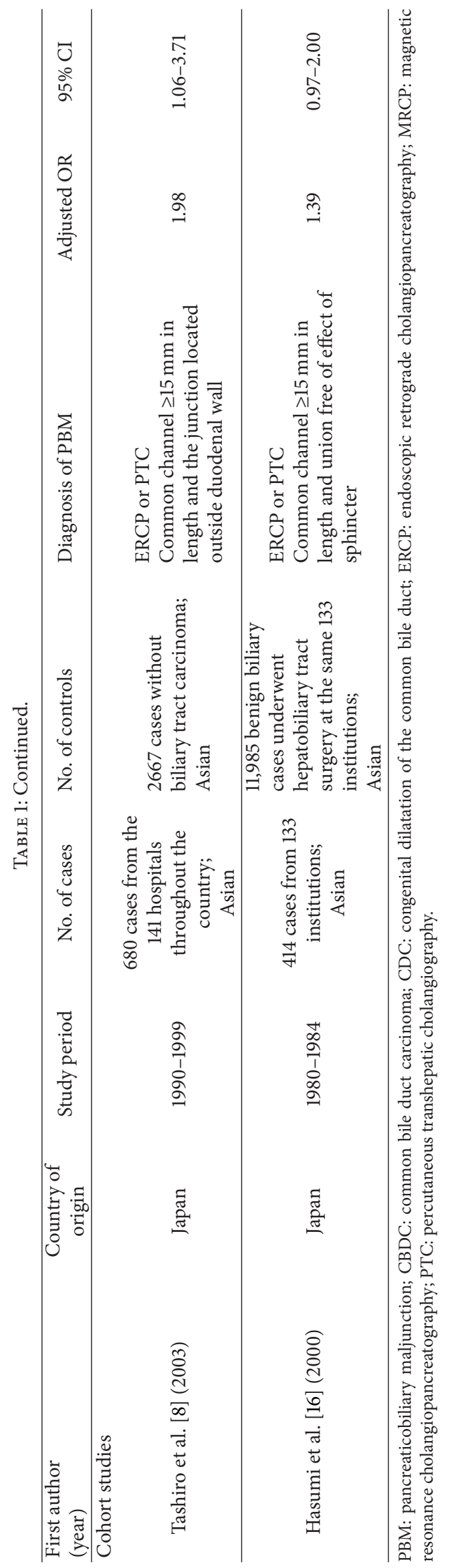


TABLE 2: The clinical data of included studies on CBDC patients with or without associated PBM.

\begin{tabular}{|c|c|c|c|c|c|c|}
\hline \multirow{2}{*}{$\begin{array}{l}\text { First author } \\
\text { (year) }\end{array}$} & \multicolumn{3}{|c|}{ With PBM } & \multicolumn{3}{|c|}{ Without PBM } \\
\hline & $\begin{array}{c}\text { Number of } \\
\text { patients }\end{array}$ & $\begin{array}{c}\text { Age at } \\
\text { diagnosis }\end{array}$ & $\begin{array}{c}\text { Incidence of } \\
\text { CDC }\end{array}$ & $\begin{array}{c}\text { Number of } \\
\text { patients }\end{array}$ & $\begin{array}{c}\text { Age at } \\
\text { diagnosis }\end{array}$ & $\begin{array}{l}\text { Incidence of } \\
\text { CDC }\end{array}$ \\
\hline Tashiro et al. [8] (2003) & 153 & $24.3 \pm 23.9$ & $29 / 153$ & 527 & $47.3 \pm 19.3$ & $61 / 527$ \\
\hline Kamisawa et al. [13] (2010) & 16 & $54.6 \pm 14.2$ & $5 / 16$ & 91 & $58.8 \pm 9.1$ & $20 / 91$ \\
\hline Matsumoto et al. [18] (2003) & 39 & $63.0 \pm 11.0$ & $12 / 39$ & 211 & $71.4 \pm 10.4$ & $24 / 211$ \\
\hline Wang et al. [23] (1998) & 11 & NA & $4 / 11$ & 16 & NA & $3 / 16$ \\
\hline Roukounakis et al. [10] (2007) & 22 & $65.6 \pm 9.8$ & $7 / 22$ & 4 & $68.0 \pm 11.5$ & $1 / 4$ \\
\hline Ohuchida et al. [24] (2006) & 55 & $30.0 \pm 20.7$ & $12 / 55$ & 141 & $52.9 \pm 16.8$ & $11 / 141$ \\
\hline Hasumi et al. [16] (2000) & 63 & $53.2 \pm 11.9$ & $8 / 63$ & 351 & $66.5 \pm 18.4$ & $44 / 351$ \\
\hline Suda et al. [26] (1983) & 27 & NA & NA & 45 & NA & NA \\
\hline Song et al. [25] (1999) & 38 & $36.5 \pm 14.1$ & $7 / 38$ & 291 & $49.2 \pm 12.2$ & $15 / 291$ \\
\hline Seki et al. [27] (2005) & 6 & $51.6 \pm 13.3$ & $4 / 6$ & 32 & $50.9 \pm 13.7$ & $3 / 32$ \\
\hline
\end{tabular}

PBM: pancreaticobiliary maljunction; CBDC: common bile duct carcinoma; CDC: congenital dilatation of the common bile duct; NA: not available.

did not provide the incidence of CDC in CBDC patients with PBM.

3.3. Meta-Analysis Results. The OR estimates for the association between PBM and CBDC are shown in Figure 2(a). The $\mathrm{OR}$ estimates from eight case-control studies $(\mathrm{OR}=1.41$, 95\% CI: 1.10-1.81), two cohort studies (OR $=1.52,95 \%$ CI: 1.11-2.07), and all studies combined ( $\mathrm{OR}=1.45,95 \% \mathrm{CI}$ : 1.19-1.76) were statistically significant and showed a positive association with PBM. The pooled incidence of PBM was 6.73\% (144 of 2139) in CBDC patients and 5.10\% (1223 of $23967)$ in controls. The fixed-effects model was used because the test for heterogeneity was not statistically significant $(P=$ $0.43, I^{2}=0.9 \%$ ).

Diagnosis age in each study was extracted as mean \pm SD (Table 2). The Forest plot for mean age at diagnosis in CBDC patients with or without PBM was displayed in Figure 2(b). There is no substantial heterogeneity $\left(P=0.35, I^{2}=10.1 \%\right)$ and the fixed-effects model was used. The OR were -0.62 ( $95 \%$ CI: -0.95 to -0.29 ) from the six case-control studies, -0.39 (95\% CI: -0.61 to -0.17 ) from the two cohort studies, and -0.46 (95\% CI: -0.64 to -0.28 ) overall, which revealed that the mean age at diagnosis for CBDC in PBM patients was younger than that of patients without PBM.

Overall there was no heterogeneity amongst the 9 studies (seven case-control studies and 2 cohort studies) when examining the incidence of CDC in CBDC patients with or without PBM $\left(P=0.13, I^{2}=36.6 \%\right)$ and the fixedeffects model was used (Table 2). The Forest plot was shown in Figure 2(c): the OR estimate from the seven case-control studies was 3.20 (95\% CI: 2.08-4.92) and from the two cohort studies was 1.52 (95\% CI: 1.01-2.29) and the overall estimate was 2.14 (95\% CI: 1.60-2.87). The results suggest that there is a positive correlation between the increased risk of CBDC and the incidence of $\mathrm{CDC}$ with PBM.

3.4. Publication Bias. Figure 3 showed that no evidence of publication bias was found from both visualization of Begg's funnel plot and Egger's test. $P$ values for Begg's adjusted rank correlation test and Egger's regression asymmetry test were 0.858 and 0.506 for the association of PBM and CBDC risk, respectively.

\section{Discussion}

The results of this meta-analysis based on 10 studies (8 casecontrol studies and 2 cohort studies) provide supportive evidence of a positive association between PBM and CBDC. We could not detect any important difference between casecontrol and cohort studies, which provides support to the positive association observed.

PBM is an important carcinogenic factor in biliary tract carcinoma, especially in Asian patients. Many researches have been published to reveal a potential association between PBM and the development of CBDC; however, the association remains controversial. Tashiro et al. [8] and Tanaka et al. [28] reported that the number of bile duct cancers exceeded the number of gallbladder cancers in the dilated bile duct cases of PBM, while gallbladder cancer frequently occurred but bile duct carcinoma rarely occurred in PBM cases not associated with dilatation. Whereas Seki et al. [27] demonstrated that bile duct cancer did not occur in cystic dilatation, $50 \%$ of bile duct cancers developed from nondilated bile duct epithelium with PBM over 50 years old. The results from our metaanalysis indicate that patients with PBM have an increased risk of developing CBDC compared with those without PBM, especially those PBM patients with CDC.

One recent published meta-analysis has assessed PBM and the risk of developing gallbladder carcinoma. Deng et al. [15] combined six studies to investigate the difference in the incidence of PBM between gallbladder cancer patients and controls. The results indicated that the incidence of PBM among gallbladder cancer patients is significantly higher than that in general population. The combined OR was 7.41 (95\% CI: 5.03-10.77). However, this study did not demonstrate the relationship between PBM and CBDC. As the CBDC is the common carcinoma associated with PBM, we consider that it is beneficial to evaluate the correlation between PBM and $\mathrm{CBDC}$ in a comprehensive analysis. 


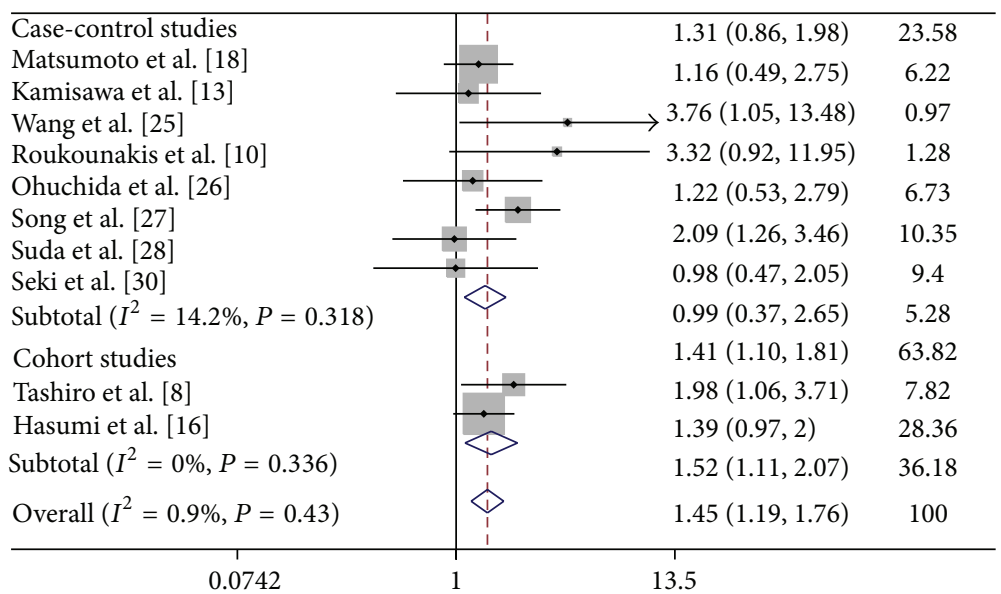

(a)

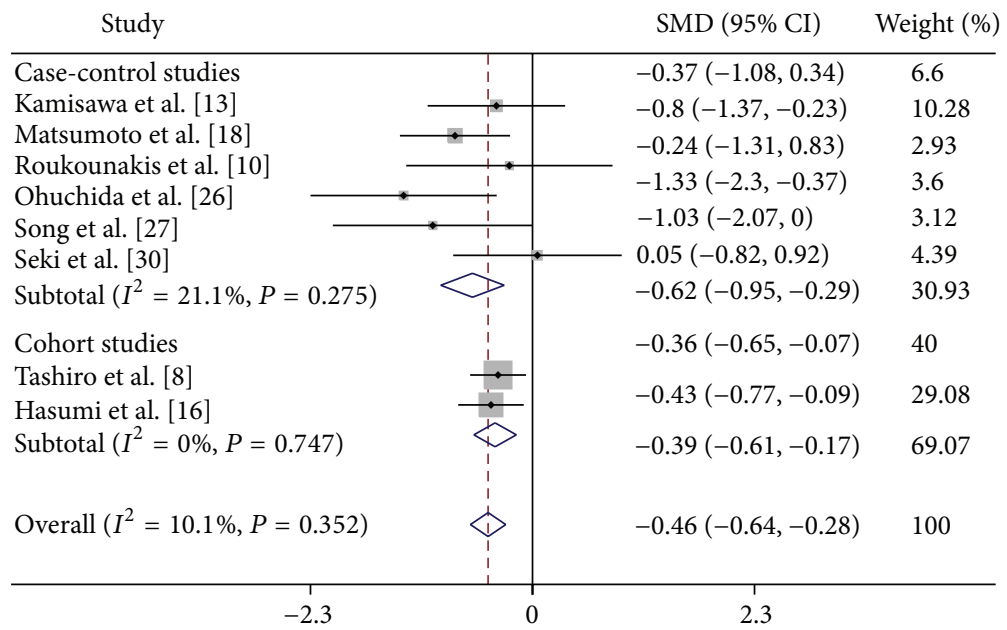

(b)

\begin{tabular}{|c|c|c|c|}
\hline Study & & SMD (95\% CI) & Weight (\% \\
\hline Case-control studies & & $1.61(0.5,5.19)$ & 7.63 \\
\hline Kamisawa et al. [13] & $\cdot$ & $3.46(1.55,7.72)$ & 9.62 \\
\hline Matsumoto et al. [18] & $\frac{1}{1 \cdot}$ & $2.48(0.43,14.34)$ & 2.89 \\
\hline Wang et al. [25] & $\overrightarrow{1}$ & $1.4(0.12,15.97)$ & 2.14 \\
\hline $\begin{array}{l}\text { Roukounakis et al. [10] } \\
\text { Ohuchida et al. [26] }\end{array}$ & $\frac{1}{1}$ & $3.3(1.36,8.01)$ & 8.95 \\
\hline Song et al. [27] & $\frac{1}{1+2}$ & $4.15(1.57,10.97)$ & 5.24 \\
\hline Seki et al. [30] & & $19.33(2.43,153.55)$ & 0.59 \\
\hline Subtotal $\left(I^{2}=0 \%, P=0.536\right)$ & $>$ & $3.2(2.08,4.92)$ & 37.05 \\
\hline \multicolumn{4}{|l|}{ Cohort studies } \\
\hline Tashiro et al. [8] & 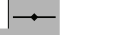 & $1.79(1.1,2.9)$ & 41.27 \\
\hline Hasumi et al. [16] & T & $1.01(0.45,2.27)$ & 21.68 \\
\hline Subtotal $\left(I^{2}=28.2 \%, P=0.238\right)$ & 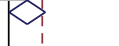 & $1.52(1.01,2.29)$ & 62.95 \\
\hline Overall $\left(I^{2}=36.6 \%, P=0.126\right)$ & \langle & $2.14(1.60,2.87)$ & 100 \\
\hline 0.00651 & 1 & 154 & \\
\hline
\end{tabular}

(c)

FIGURE 2: Forest plot of the association between PBM and CBDC risk. (a) Summary risk of CBDC associated with PBM. (b) Forest plot describing the SMD for diagnosis age in CBDC patients with or without PBM. (c) Forest plot describing the OR of the incidence of CDC in CBDC patients with or without PBM. PBM: pancreaticobiliary maljunction; CBDC: common bile duct carcinoma; SMD: standard mean difference; CI: confidence interval; OR: odds ratio. 


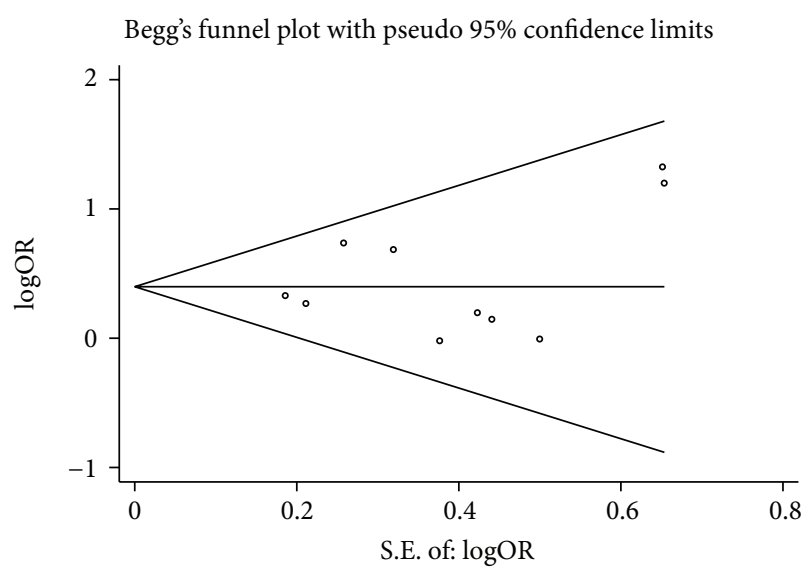

FIGURE 3: Funnel plot of studies evaluating the relationships between PBM and risk of CBDC. PBM: pancreaticobiliary maljunction; CBDC: common bile duct carcinoma; S.E.: standard error; OR: odds ratio.

Several mechanisms have been proposed to potentially underlie the development of CBDC in individuals with $\mathrm{PBM}$. The carcinogenesis of CBDC coexisting with PBM is considered to involve the hyperplasia-dysplasia-carcinoma sequence provoked by the chronic inflammation that is a result of the reflux of pancreatic juices into the biliary tract $[11,29]$. Strong cytotoxic substances (such as lysolecithin, secondary bile acids, and deconjugated bile acids) are produced when phospholipase $\mathrm{A} 2$ in the pancreatic juice mixes with bile, which are recognized clinically and experimentally to be injurious to cell membranes, and as a result, the chronic inflammation provokes high proliferative activity in the mucosal epithelia, finally causing CBDC in PBM patients [30-32]. The carcinogenesis of common bile duct epithelial cells in PBM patients is a multigene pathological process, associated with many genetic mutations, such as K-ras and p53 [33-35]. In addition, pancreatic juice can easily flow into the common bile duct in C-P type in which the common bile duct joins the pancreatic duct at approximately $90^{\circ}$. This mechanism is thought to be different from the adenomacarcinoma sequence or the de novo carcinogenesis associated with biliary tract cancer in the population without PBM [3].

PBM can provide stagnant sites exposed to a mixture of bile and pancreatic juice over a prolonged period. Prolonged exposure causes persistent chronic inflammation in the biliary lining epithelium, leading to hyperplasia, atypia, and ultimately carcinoma [36]. This suggests that the stagnant site is an indispensable factor for carcinogenesis. Such sites could be provided by the dilated cyst in patients with PBM accompanied by CDC, and canceration is more likely to occur within a dilated cyst. Interestingly, Kosaka et al. [37] reported that it is extremely common for $\mathrm{H}$. bilis to colonize the biliary system in patients with PBM. Pathophysiological alterations, such as erosion, desquamation, hyperplasia, and gastric or intestinal metaplasia [17], along with bile stasis, may allow $H$. bilis to colonize the biliary system in PBM. H. bilis may play an important role in inflammation-associated biliary carcinogenesis.
The present meta-analysis must be interpreted in the context of several limitations: (1) 8 of 10 studies included in this meta-analysis used a case-control design, which is more susceptible to recall and selection biases than a cohort design; the other 2 cohort studies may be affected by detection bias as patients with PBM are under increased medical examination because of clinical symptoms such as abdominal pain, vomiting, jaundice, and fever and thus may be more likely to be diagnosed with CBDC. These biases may confuse the true association between PBM and CBDC. (2) The definition of PBM with the length of common channel varied across studies ( $>8 \mathrm{~mm}, 10 \mathrm{~mm}$, or $15 \mathrm{~mm}$ ) and this restricts the comparability of these studies. Therefore, some degree of inappropriate diagnosis of PBM is likely to occur. These nonuniform diagnostic criteria would tend to reduce the magnitude of the results. (3) Some subgroup analyses were based on few studies and the results need to be cautiously interpreted. Moreover, confounding is also likely to be present. Although most studies controlled for some confounding factors, such as smoking, and alcohol abuse, which have generally been associated with an increased risk of $\mathrm{CBDC}$, we cannot exclude the possibility of residual confounding. (4) Other potential limitations of our study could be due to the language restriction in that we only selected articles published in English. (5) Finally, as in any meta-analysis, the possibility of publication bias is of concern, because small studies with null results tend not to be published. However, the results obtained from funnel plot analysis and formal statistical tests did not provide evidence for such bias.

In summary, this meta-analysis suggests an association between PBM and the risk of CBDC, especially for relatively young patients with CDC. However, to strengthen our findings, well-designed prospective studies with accurate diagnostic criteria of PBM may help to explore the relation between PBM and the risk of CBDC.

\section{Conflict of Interests}

The authors declare that they have no conflict of interests.

\section{Acknowledgment}

The authors are indebted to the authors of the primary studies.

\section{References}

[1] S. M. Hong, M. J. Kim, D. Y. Pi et al., "Analysis of extrahepatic bile duct carcinomas according to the new American joint committee on cancer staging system focused on tumor classification problems in 222 patients," Cancer, vol. 104, no. 4, pp. 802-810, 2005.

[2] J. N. Vauthey and L. H. Blumgart, "Recent advances in the management of cholangiocarcinomas," Seminars in Liver Disease, vol. 14, no. 2, pp. 109-114, 1994.

[3] H. Malhi and G. J. Gores, "Cholangiocarcinoma: modern advances in understanding a deadly old disease," Journal of Hepatology, vol. 45, no. 6, pp. 856-867, 2006. 
[4] S. Miyakawa, S. Ishihara, A. Horiguchi, T. Takada, M. Miyazaki, and T. Nagakawa, "Biliary tract cancer treatment: 5,584 results from the biliary tract cancer statistics registry from 1998 to 2004 in Japan," Journal of Hepato-Biliary-Pancreatic Surgery, vol. 16, no. 1, pp. 1-7, 2009.

[5] S. A. Khan, H. C. Thomas, B. R. Davidson, and S. D. TaylorRobinson, "Cholangiocarcinoma," The Lancet, vol. 366, no. 9493, pp. 1303-1314, 2005.

[6] Y. Shaib and H. B. El-Serag, "The epidemiology of cholangiocarcinoma," Seminars in Liver Disease, vol. 24, no. 2, pp. 115-125, 2004.

[7] M. T. Carriaga and D. E. Henson, "Liver, gallbladder, extrahepatic bile ducts, and pancreas," Cancer, vol. 75, no. 1, supplement, pp. 171-190, 1995.

[8] S. Tashiro, T. Imaizumi, H. Ohkawa et al., "Pancreaticobiliary maljunction: retrospective and nationwide survey in Japan," Journal of Hepato-Biliary-Pancreatic Surgery, vol. 10, no. 5, pp. 345-351, 2003.

[9] M. Seki, A. Yanagisawa, E. Ninomiya et al., "Clinicopathology of pancreaticobiliary maljunction: relationship between alterations in background biliary epithelium and neoplastic development," Journal of Hepato-Biliary-Pancreatic Surgery, vol. 12, no. 3, pp. 254-262, 2005.

[10] N. Roukounakis, S. Manolakopoulos, D. Tzourmakliotis, S. Bethanis, T. M. McCarty, and J. Cuhn, "Biliary tract malignancy and abnormal pancreaticobiliary junction in a western population," Journal of Gastroenterology and Hepatology, vol. 22, no. 11, pp. 1949-1952, 2007.

[11] T. Funabiki, T. Matsubara, S. Miyakawa, and S. Ishihara, "Pancreaticobiliary maljunction and carcinogenesis to biliary and pancreatic malignancy," Langenbeck's Archives of Surgery, vol. 394, no. 1, pp. 159-169, 2009.

[12] T. Kamisawa, H. Ando, M. Suyama et al., "Japanese clinical practice guidelines for pancreaticobiliary maljunction," Journal of Gastroenterology, vol. 47, no. 7, pp. 731-759, 2012.

[13] T. Kamisawa, M. Suyama, N. Fujita et al., "Pancreatobiliary reflux and the length of a common channel," Journal of HepatoBiliary-Pancreatic Sciences, vol. 17, no. 6, pp. 865-870, 2010.

[14] M. Miyazaki, T. Takada, S. Miyakawa et al., "Risk factors for biliary tract and ampullary carcinomas and prophylactic surgery for these factors," Journal of Hepato-Biliary-Pancreatic Surgery, vol. 15, no. 1, pp. 15-24, 2008.

[15] Y. L. Deng, N. S. Cheng, Y. X. Lin et al., "Relationship between pancreaticobiliary maljunction and gallbladder carcinoma: a meta-analysis," Hepatobiliary and Pancreatic Diseases International, vol. 10, no. 6, pp. 570-580, 2011.

[16] A. Hasumi, H. Matsui, A. Sugioka et al., "Precancerous conditions of biliary tract cancer in patients with pancreaticobiliary malfunction: reappraisal of nationwide survey in Japan," Journal of Hepato-Biliary-Pancreatic Surgery, vol. 7, no. 6, pp. 551-555, 2000.

[17] M. Nagai, M. Watanabe, T. Iwase, K. Yamao, and S. Isaji, "Clinical and genetic analysis of noncancerous and cancerous biliary epithelium in patients with pancreaticobiliary maljunction," World Journal of Surgery, vol. 26, no. 1, pp. 91-98, 2002.

[18] Y. Matsumoto, H. Fujii, J. Itakura et al., "Pancreaticobiliary maljunction: pathophysiological and clinical aspects and the impact on biliary carcinogenesis," Langenbeck's Archives of Surgery, vol. 388, no. 2, pp. 122-131, 2003.

[19] A. Liberati, D. G. Altman, J. Tetzlaff et al., "The PRISMA statement for reporting systematic reviews and meta-analyses of studies that evaluate healthcare interventions: explanation and elaboration," The British Medical Journal, vol. 339, article b2700, 2009.

[20] S. Greenland and M. P. Longnecker, "Methods for trend estimation from summarized dose-response data, with applications to meta-analysis," American Journal of Epidemiology, vol. 135, no. 11, pp. 1301-1309, 1992.

[21] C. B. Begg and M. Mazumdar, "Operating characteristics of a rank correlation test for publication bias," Biometrics, vol. 50, no. 4, pp. 1088-1101, 1994.

[22] M. Egger, G. D. Smith, M. Schneider, and C. Minder, "Bias in meta-analysis detected by a simple, graphical test," The British Medical Journal, vol. 315, no. 7109, pp. 629-634, 1997.

[23] H. P. Wang, M. S. Wu, C. C. Lin et al., "Pancreaticobiliary diseases associated with anomalous pancreaticobiliary ductal union," Gastrointestinal Endoscopy, vol. 48, no. 2, pp. 184-189, 1998.

[24] J. Ohuchida, K. Chijiiwa, M. Hiyoshi, K. Kobayashi, H. Konomi, and M. Tanaka, "Long-term results of treatment for pancreaticobiliary maljunction without bile duct dilatation," Archives of Surgery, vol. 141, no. 11, pp. 1066-1070, 2006.

[25] H. K. Song, M. H. Kim, S. J. Myung et al., "Choledochal cyst associated the with anomalous union of pancreaticobiliary duct (AUPBD) has a more grave clinical course than choledochal cyst alone," The Korean Journal of Internal Medicine, vol. 14, no. 2, pp. 1-8, 1999.

[26] K. Suda, T. Miyano, I. Konuma, and M. Matsumoto, "An abnormal pancreatico-choledocho-ductal junction in cases of biliary tract carcinoma," Cancer, vol. 52, no. 11, pp. 2086-2088, 1983.

[27] M. Seki, A. Yanagisawa, E. Ninomiya et al., "Clinicopathology of pancreaticobiliary maljunction: relationship between alterations in background biliary epithelium and neoplastic development," Journal of Hepato-Biliary-Pancreatic Surgery, vol. 12, no. 3, pp. 254-262, 2005.

[28] S. Tanaka, M. Kubota, M. Yagi et al., "An 11-year-old male patient demonstrating cholangiocarcinoma associated with congenital biliary dilatation," Journal of Pediatric Surgery, vol. 41, no. 1, pp. e15-e19, 2006.

[29] K. Shimada, J. Yanagisawa, and F. Nakayama, "Increased lysophosphatidylcholine and pancreatic enzyme content in bile of patients with anomalous pancreaticobiliary ductal junction," Hepatology, vol. 13, no. 3, pp. 438-444, 1991.

[30] A. Tsuchida and T. Itoi, "Carcinogenesis and chemoprevention of biliary tract cancer in pancreaticobiliary maljunction," World Journal of Gastrointestinal Oncology, vol. 2, no. 3, pp. 130-135, 2010.

[31] D. Qian, T. Kinouchi, K. Kunitomo et al., "Mutagenicity of the bile of dogs with an experimental model of an anomalous arrangement of the pancreaticobiliary duct," Carcinogenesis, vol. 14, no. 4, pp. 743-747, 1993.

[32] T. Funabiki, K. Sugiue, T. Matsubara, H. Amano, and M. Ochiai, "Bile acids and biliary carcinoma in pancreaticobiliary maljunction," Keio Journal of Medicine, vol. 40, no. 3, pp. 118122, 1991.

[33] S. Masuhara, K. Kasuya, T. Aoki, A. Yoshimatsu, A. Tsuchida, and Y. Koyanagi, "Relation between K-ras codon 12 mutation and $\mathrm{p} 53$ protein overexpression in gallbladder cancer and biliary ductal epithelia in patients with pancreaticobiliary maljunction," Journal of Hepato-Biliary-Pancreatic Surgery, vol. 7, no. 2, pp. 198-205, 2000. 
[34] T. Matsubara, Y. Sakurai, Y. Sasayama et al., "K-ras point mutations in cancerous and noncancerous biliary epithelium in patients with pancreaticobiliary maljunction," Cancer, vol. 77, no. 8, pp. 1752-1757, 1996.

[35] T. Matsubara, Y. Sakurai, L. Z. Zhi, H. Miura, M. Ochiai, and T. Funabiki, "K-ras and p53 gene mutations in noncancerous biliary lesions of patients with pancreaticobiliary maljunction," Journal of Hepato-Biliary-Pancreatic Surgery, vol. 9, no. 3, pp. 312-321, 2002.

[36] K. Suda, T. Miyano, and K. Hashimoto, "The choledochopancreatico-ductal junction in infantile obstructive jaundice diseases," Acta Pathologica Japonica, vol. 30, no. 2, pp. 187-194, 1980.

[37] T. Kosaka, Y. Tajima, T. Kuroki et al., "Helicobacter bilis colonization of the biliary system in patients with pancreaticobiliary maljunction," The British Journal of Surgery, vol. 97, no. 4, pp. 544-549, 2010. 


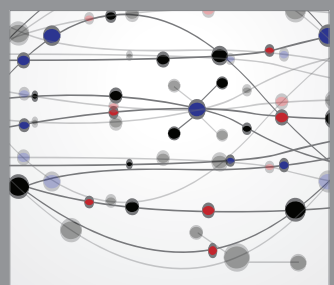

The Scientific World Journal
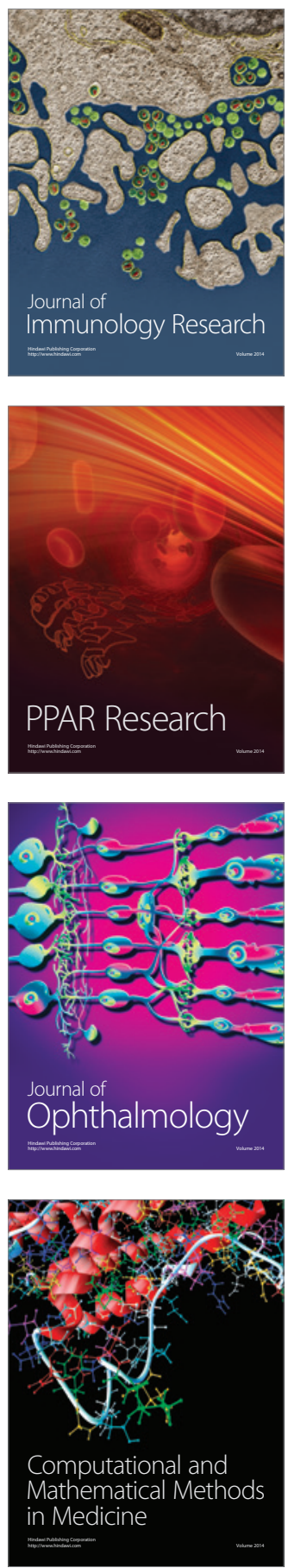

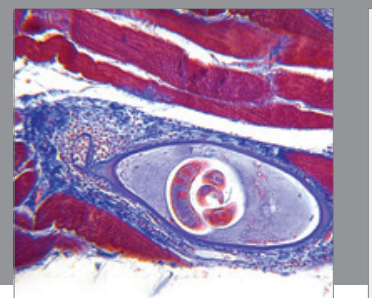

Gastroenterology

Research and Practice
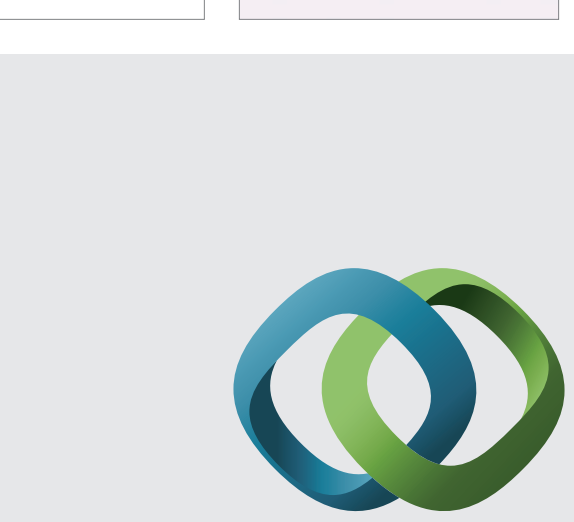

\section{Hindawi}

Submit your manuscripts at

http://www.hindawi.com
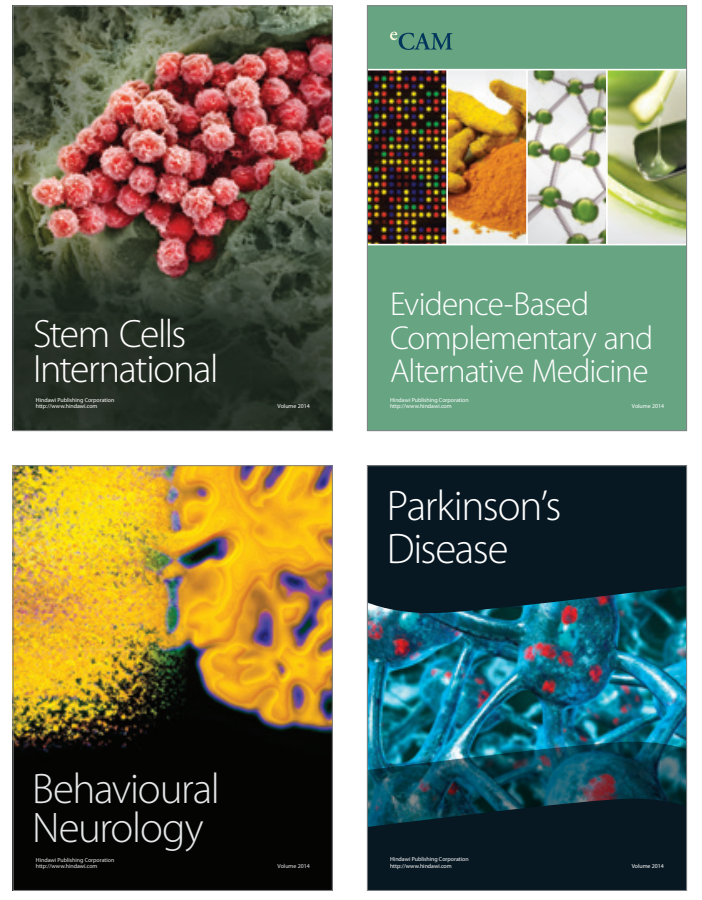
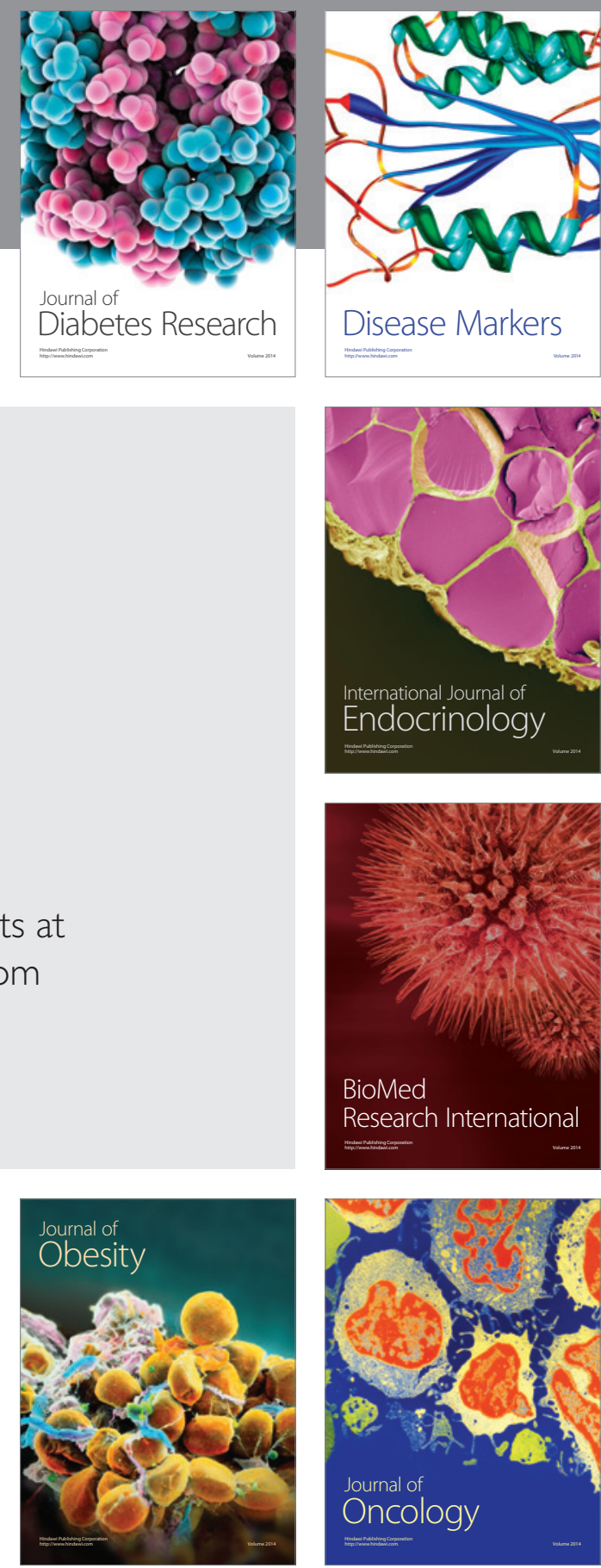

Disease Markers
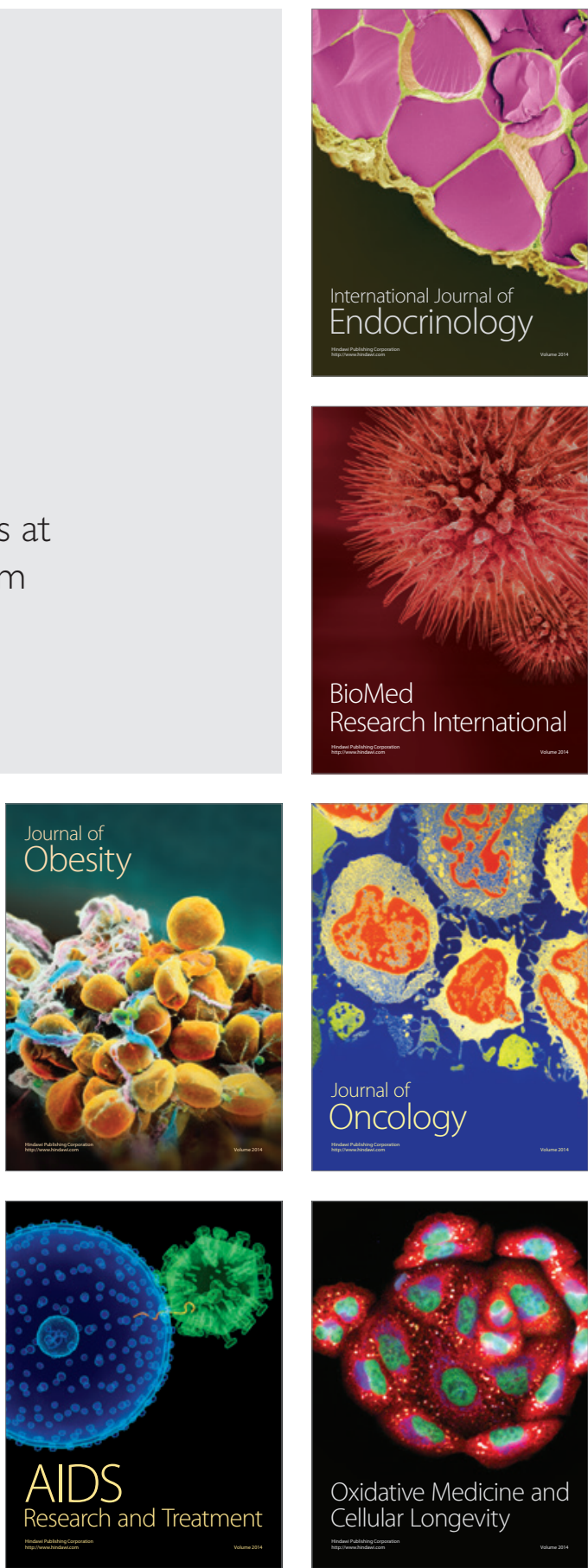